\title{
High-resolution spectroscopy of flat-spectrum quasars ${ }^{\star}$
}

\section{Redshifted emission and absorption in PKS 2145+067}

\author{
A. Tappe and J. H. Black
}

\author{
Chalmers Centre for Astrophysics and Space Science, Onsala Space Observatory, Chalmers University of Technology, \\ 43992 Onsala, Sweden \\ e-mail: [achim;jblack]@oso.chalmers.se
}

Received 13 December 2003 / Accepted 24 May 2004

\begin{abstract}
We report Very Large Telescope (VLT) observations of the quasar PKS 2145+067. We obtained high-resolution $\left(R \approx 100000\right.$ or $\left.3 \mathrm{~km} \mathrm{~s}^{-1}\right)$ optical spectra with the UV-Visual Echelle Spectrograph (UVES) at the VLT UT2 telescope. The emission redshift measured from [O $\mathrm{III}]$ is $z_{\mathrm{em}}=1.0003$. In our spectra, the well-known intervening metal-rich absorption system at $z_{\text {abs }}=0.79089$ shows $\mathrm{Mg}$ II absorption lines resolved into 15 velocity components with a total spread of about $206 \mathrm{~km} \mathrm{~s}^{-1}$. This absorption is consistent with a line of sight passing through the extended gaseous halo of a disk galaxy. The derived Doppler line broadening parameters are in the range $b=2$ to $5 \mathrm{~km} \mathrm{~s}^{-1}$. We also detected absorption lines of $\mathrm{Mg}_{\mathrm{I}}, \mathrm{Fe}_{\mathrm{II}}$, and $\mathrm{Ca}_{\text {II }}$ in the same system. The ionization state of the absorbing gas is analyzed and compared with previous studies of PKS $2145+067$. Finally, we discuss the implications of high-resolution data for proposed cosmological variations of the fine-structure constant.
\end{abstract}

Key words. galaxies: quasars: absorption lines - galaxies: quasars: emission lines galaxies: quasars: individual: PKS 2145+067

\section{Introduction}

Quasar spectroscopy has developed into a powerful multipurpose tool with the advent of 8-10 m telescopes equipped with sensitive spectrographs and the first release of the Sloan Digital Sky Survey (SDSS) data (Abazajian et al. 2003; Schneider et al. 2003). It allows the study of matter seen through emission and absorption lines of atoms and molecules up to redshifts $z \approx 6$ (Fan et al. 2003). Emission and intrinsic absorption lines probe the structure and physical properties of the emission region in Active Galactic Nuclei (Osterbrock \& Mathews 1986; Sulentic et al. 2000; Véron-Cetty \& Véron 2000). Intervening absorption lines are used to study the kinematics, chemical composition, and ionization state of gas associated with galaxies at intermediate redshift (e.g. Steidel \& Sargent 1992; Churchill \& Vogt 2001; Churchill et al. 2003; Ding et al. 2003). Galactic absorption lines seen in quasar spectra offer unbiased lines of sight through the Galactic interstellar medium in contrast to traditional studies using OB-type stars as background sources; OB stars are concentrated in the Galactic plane and associated directly with interstellar matter, whereas quasars reside far outside the Milky Way with no physical connection to the absorbing material. Quasar background sources offer opportunities for complementary spectroscopy in the

* Based on observations collected at the European Southern Observatory, Paranal, Chile (ESO Programmes 67.C-0157 and 70.C-0239). radio, mm, and X-ray domains for the same line of sight. Finally, fine-structure splittings observed in quasar emission and absorption lines are used to constrain the possible space/time-variation of the fine-structure constant $\alpha$ (Bahcall et al. 2004; Murphy et al. 2003a).

We have studied a sample of 9 flat-spectrum quasars with the VLT/UVES (Very Large Telescope; UV-Visual Echelle Spectrograph) at high resolution $\left(R \approx 100000\right.$ or $3 \mathrm{~km} \mathrm{~s}^{-1}$ on the brighter, $R \approx 50000$ on the fainter sources). We selected the quasars according to the following criteria: bright enough for high-resolution spectroscopy with UVES (visual magnitude brighter than $18.5 \mathrm{mag}$ ); nearly featureless continuum with strong mm-wavelength and X-ray fluxes; low Galactic latitude and/or known Galactic absorption at mm-wavelengths. Background sources with such properties allow the measurement of Galactic interstellar absorption across the electromagnetic spectrum from $\mathrm{mm}$ to X-ray wavelengths for the same line of sight. This is one of the main rationales in our project.

Here, we focus on the well studied quasar PKS 2145+067: its emission and continuum properties are measured (Steidel \& Sargent 1991); Hubble Space Telescope Faint Object Spectrograph (HST/FOS) spectra have been obtained within the HST quasar absorption line key project (Bahcall et al. 1993; Bergeron et al. 1994); its intervening Mg II absorption system at $z_{\mathrm{abs}}=0.79$ has been studied at a resolution of $R \approx 45000$ (Churchill \& Vogt 2001); and the associated host galaxy at $z=$ 0.79 was identified by Bergeron \& Boissé (1991). 
In this paper, we study redshifted emission and absorption in PKS 2145+067 at previously unequaled resolution. Highresolution is required to identify the individual components in complex absorption profiles, and to study the kinematics and the ionization state of the absorber in detail. We show that a factor-of-two improvement in resolution over previous work reveals new structure in $\mathrm{Mg}$ II lines, which can lead to an improved analysis of the $z_{\mathrm{abs}}=0.79$ absorbing system. We will present the Galactic absorption lines, redshifted $\mathrm{Mg}$ II absorption systems, and quasar emission lines for the complete sample of quasars elsewhere (Tappe \& Black 2004, in preparation). In Sect. 2, we describe the observations and address the data reduction and analysis techniques. We present our results in Sect. 3, and discuss the kinematics, abundances, and ionization state of the intervening $\mathrm{Mg}$ II absorber in Sect. 4. Our conclusions and a comparison with previous work are given in Sect. 5 .

\section{Observations and data analysis}

\subsection{VLT observations}

PKS 2145+067 was observed as part of a sample of 9 quasars during 2001 May 11 to June 29, and 2002 October 02 to 2003 Febuary 05 . The observations were carried out in service mode at the VLT Kueyen (UT2) telescope operated by ESO (European Southern Observatory) on Cerro Paranal, Chile. The UVES instrument was used in standard grating settings DIC2 390+860 and RED 520, with slit widths of 0'. 4 in the blue and 0,3 in the red. This combination of settings yields an almost complete wavelength coverage from 3300-10400 with nominal resolutions of about $4.3 \mathrm{~km} \mathrm{~s}^{-1}$ in the blue arm of the spectrograph, $3300-4500 \AA$, and $2.7 \mathrm{~km} \mathrm{~s}^{-1}$ at longer wavelengths (see Table 1 for a more detailed list). Two exposures of $2400 \mathrm{~s}$ duration were made in each setting.

Observations of the type presented here are ideally suited for VLT service-mode operations: the spectrograph configurations can be planned fully in advance, and the measurements can be executed when the conditions of seeing and target accessibility are satisfied. Because UVES has proven to be extremely stable, standard calibrations are adequate for this work.

\subsection{Data reduction}

The VLT pipeline processes the raw images into calibrated, one-dimensional spectra as described in the VLT White Book ${ }^{1}$. Table 1 shows a list of the UVES settings and the obtained spectra for PKS $2145+067$. The intensity scale of the spectra is a relative flux that cannot be calibrated accurately to an absolute scale because the slit widths were smaller than the atmospheric seeing-disks of the sources. The wavelength scales were established with reference to Th-Ar comparison spectra, and refer to the topocentric standard of rest in standard air in the pipeline-processed spectra.

We performed a preliminary inspection of the pipeline reduced spectra with "Spectool", a spectrum examination and

\footnotetext{
1 The VLT White book is accessible via the Paranal Observatory homepage, http://www. eso.org/paranal/
}

analysis tool with graphical user interface within the IRAF ${ }^{2}$ software system. At this stage, we converted the spectra to the heliocentric standard of rest with the IRAF "rvcorrect" and "dopcor" tasks, and combined the two exposures of each wavelength region for the identification of absorption features (see Sect. 2.3). Note that the radial velocity corrections in the original data headers were incorrect.

For further detailed analysis, we selected small wavelength intervals from each exposure, rebinned them to the same wavelength scale with the IRAF "dispcor" task using a 5th order polynomial, and then combined the rebinned intervals with the IRAF "scombine" task. In combining data of different resolution, the spectrum of higher resolution was convolved to the resolution of the coarser spectrum. Separate pieces of data were weighted by the signal-to-noise ratio. In order to enhance further the signal-to-noise for the detection of weak transitions, in particular $\mathrm{Fe}$ I, we combined several spectral intervals covering different transitions of the same ion on a common scale of velocity. In this case, each spectral interval was weighted by $1 / \lambda_{i} f_{i}$ according to the following formula for the column density $N$ in the weak-line limit for a combined spectrum,

$N\left[\mathrm{~m}^{-2}\right]=4 \pi \varepsilon_{0} \frac{m_{\mathrm{e}} c}{\pi e^{2}} \frac{1}{n} \sum_{i=1}^{n} \frac{W_{i}\left[\mathrm{~m} \mathrm{~s}^{-1}\right]}{\lambda_{i}[\mathrm{~m}] f_{i}}$,

or

$N\left[\mathrm{~cm}^{-2}\right]=3.7679 \times 10^{14} \frac{1}{n} \sum_{i=1}^{n} \frac{W_{i}\left[\mathrm{~km} \mathrm{~s}^{-1}\right]}{\lambda_{i}[\AA] f_{i}}$,

where $n$ is the total number of combined spectra of common resolution and sampling, $W_{i}$ is the equivalent width of the $i$ th spectrum in velocity units, and $\lambda_{i} f_{i}$ is the product of transition wavelength and oscillator strength.

\subsection{Data analysis}

We identified absorption features in the combined spectra (cf. Sect. 2.2) with a finding list based on atomic data compiled by Morton (1991), complemented by several transitions of molecular species. In addition, we constructed linelists for known redshifted systems from the NASA Extragalactic Database ${ }^{3}$ and from a reanalysis of HST/FOS data by Bechtold et al. (2002). We also searched for weak absorption lines with redshifts close to the emission redshift of PKS 2145+067.

"Spectool" allows to mark the line positions of several color-coded linelists in the spectra. It can also display the original uncombined exposures as well as error and stellar reference spectra simultaneously, which aids greatly in the identification of spectral features. In order to identify telluric lines, we used a stellar reference spectrum of HDE 312980, which we obtained with identical spectrograph settings. Furthermore, we made use

\footnotetext{
2 IRAF (Image Reduction and Analysis Facility) and "Spectool" are maintained by the IRAF programming group at the National Optical Astronomy Observatory (NOAO) in Tucson, Arizona.

3 The NASA/IPAC Extragalactic Database (NED) is operated by the Jet Propulsion Laboratory, California Institute of Technology, under contract with the USA National Aeronautics and Space Administration.
} 
Table 1. UVES settings and obtained spectra for PKS 2145+067. The final resolution stated for both exposures was measured as part of the VLT pipeline quality control. We list the approximate signal-to-noise ratio near the center of the covered wavelength region as measured from a non-weighted combination of the two exposures.

\begin{tabular}{lcccc}
\hline \hline Wavelength region & UVES setting & Slit width & Final resolution & Approximate $S / N$ \\
\hline $3290-4515 \AA$ & DIC2 390+860, blue arm & $0 . \prime 4$ & $3.6 / 3.7 \mathrm{~km} \mathrm{~s}^{-1}$ & 10 \\
\hline $4170-5160 \AA$ & RED 520 & 0.3 & $2.9 / 3.0 \mathrm{~km} \mathrm{~s}^{-1}$ & 20 \\
$5230-6210 \AA$ & RED 520 & 0.3 & $3.3 / 3.3 \mathrm{~km} \mathrm{~s}^{-1}$ & 25 \\
\hline $6700-8515 \AA$ & DIC2 390+860, red arm & $0 . \prime 3$ & $2.6 / 2.7 \mathrm{~km} \mathrm{~s}^{-1}$ & 20 \\
$8655-10425 \AA$ & DIC2 390+860, red arm & 0.3 & $3.1 / 3.3 \mathrm{~km} \mathrm{~s}^{-1}$ & 10 \\
\hline
\end{tabular}

of the HITRAN $2000^{4}$ database to create independent linelists for the identification of telluric water and oxygen lines.

\subsubsection{Determination of absorption properties}

The apparent line-shape of an observed absorption line is formally given by the convolution of the intrinsic line-shape and the instrumental function $\Phi(\lambda)$,

$I_{\text {obs }}(\lambda)=\Phi(\lambda) \otimes\left[I_{0}(\lambda) \mathrm{e}^{-\tau(\lambda)}\right]$,

where $I_{\mathrm{obs}}(\lambda)$ is the observed intensity, $I_{0}(\lambda)$ is the continuum intensity, and $\tau(\lambda)$ is the optical depth.

The wavelength dependence of the optical depth in Eq. (3) can be described by a Voigt function, a convolution of a Lorentzian and a Gaussian. Voigt profile fitting provides a useful method for parameterizing the absorption properties of individual components in blended absorption features given sufficient spectral resolution (cf. Churchill et al. 2003). The measured redshifts/velocities, Doppler parameters, and column densities, can be interpreted in terms of the physical nature of the absorbing gas.

The Gaussian component has a half-width at halfmaximum

$\Delta \lambda_{\mathrm{D}}=\frac{\lambda_{0}}{c}\left(\frac{2 k T}{m_{\mathrm{ion}}} \ln 2\right)^{1 / 2}=\frac{\lambda_{0}}{c}(\ln 2)^{1 / 2} b$,

where $b$ is the Doppler broadening parameter for a gas of temperature $T$ and an ion of mass $m_{\text {ion }}$. If the gas motions are microscopic thermal motions, then $T$ is the kinetic temperature, $T_{\mathrm{k}}$. If there are other gas motions, such as turbulence, then the Doppler parameter can be expressed as a sum of thermal and turbulent parts, $b=b_{\text {therm }}+b_{\text {turb }}=\left(2 k T / m_{\text {ion }}\right)^{1 / 2}+b_{\text {turb }}$, and $T$ is an upper limit on the kinetic temperature.

We used the program vPFIT (Carswell et al., see http://www.ast.cam.ac.uk/ rfc/vpfit.html; Webb 1987; Cooke 1994) to fit Voigt line-shape functions to the absorption lines (see Eq.(3)). We selected the sections of the spectrum containing absorption features (cf. Sect. 2.2), and fitted a low-order Legendre polynomial to the continuum using "Spectool". Any strong absorption or emission in these

\footnotetext{
${ }^{4}$ The high-resolution transmission molecular absorption database (HITRAN) is developed at the Atomic and Molecular Physics Division, Harvard-Smithsonian Center for Astrophysics, http://cfa-www.harvard.edu/HITRAN/
}

sections was disregarded for the continuum fitting through setting a proper threshold.

Furthermore, we used the program vPGuEss ${ }^{5}$ to set up initial guesses for the Voigt profile parameters, i.e. redshift, $b$-parameter, and column density. VPFIT then applies an iterative $\chi^{2}$-minimization routine to optimize the parameters of the initial guess. After converging, it generates a list of output parameters together with their fitting errors. These errors may or may not be meaningful, depending mainly on the $1 \sigma$ error array that comes with the observed spectra, the way the spectra are processed, the intrinsic non-uniqueness of the Voigt fit, and the amount of saturation and blending in the fitted lines. These factors were dealt with as well as possible, in particular by providing VPFIT with the proper ratio of the mean error to the measured root mean square (RMS) noise in that part of the spectrum (cf. the online manual of VPFIT about error estimates).

For the final data analysis, we referred to the latest revision of atomic data for resonance lines by Morton (2003). All redshifts quoted here refer to a vacuum heliocentric wavelength scale. Parts of the data were analyzed with an independent profile-fitting program and continuum-fitting procedure: derived line parameters were the same within the estimated uncertainties.

\section{Results}

We identified several emission and absorption features in the high resolution spectrum of PKS $2145+067$. The observed quasar emission lines include $\left.\mathrm{Mg}_{\mathrm{II}}, \mathrm{C}_{\mathrm{III}}\right]$, [O $\left.\mathrm{II}\right]$, [O III], $\mathrm{H} \beta$, and weak [Ne III] and [Ne IV], as summarized in Table 2. [Nev] 3427 is blended with a strong instrumental artifact and therefore not included here. The emission lines are extremely broad compared to the narrow absorption features, with full-width at half-maximum $(F W H M)$ between $\Delta V_{F W H M}^{\text {obs }}=600$ and $6000 \mathrm{~km} \mathrm{~s}^{-1}$. The emission redshifts obtained from a onecomponent Gaussian fit have an average of $\left\langle z_{\mathrm{em}}\right\rangle=1.001$, which is in good agreement with previous measurements (Steidel \& Sargent 1991).

Analysis of quasar emission lines can provide valuable information on the structure and physical properties of the emission region, but to perform such an analysis is beyond the scope of this work. We note here that the equivalent widths

\footnotetext{
5 vPGUEss is a graphical interface to vPFIT written by Jochen Liske, http://www.roe.ac.uk/ jol/vpguess/
} 
Table 2. Observed emission lines in PKS 2145+067.

\begin{tabular}{|c|c|c|c|c|c|}
\hline Ion & $\begin{array}{c}\lambda_{\text {vac, rest }} \\
(\AA)\end{array}$ & $\begin{array}{c}\lambda_{\text {vac, obs }}^{1)} \\
(\AA)\end{array}$ & $\begin{array}{l}\Delta V_{F W H M}^{\mathrm{obs}} \\
\left(\mathrm{km} \mathrm{s}^{-1}\right)\end{array}$ & $z_{\mathrm{em}}$ & $\begin{array}{r}W_{\text {obs }} \\
(\AA)\end{array}$ \\
\hline C III] & 1908.73 & 3819.05 & 5896 & 1.0008 & 24.4 \\
\hline \multicolumn{2}{|c|}{ broad component ${ }^{2)}$} & 3817.83 & 6765 & 1.0002 & 23.4 \\
\hline \multicolumn{2}{|c|}{ narrow component ${ }^{2)}$} & 3822.13 & 1359 & 1.0024 & 2.0 \\
\hline$[\mathrm{Ne}$ IV $]$ & 2422.87 & 4847.05 & 1421 & 1.0005 & 1.3 \\
\hline $\mathrm{Mg}_{\text {II }}$ & 2798.74 & 5605.06 & 3162 & 1.0027 & 16.5 \\
\hline [O II $]$ & 3727.66 & $7457.88^{3)}$ & 1017 & 1.0007 & 4.1 \\
\hline [Ne III] & 3869.85 & $7739.42^{3)}$ & 1098 & 0.9999 & 3.7 \\
\hline$[\mathrm{Ne}$ III $]$ & 3968.59 & $7938.28^{4)}$ & 1806 & 1.0003 & 5.1 \\
\hline $\mathrm{H} \beta$ & 4862.67 & 9725.91 & 2705 & 1.0001 & 52.7 \\
\hline [O III] & 4960.30 & $9919.36^{3), 5)}$ & 634 & 0.9997 & 16.8 \\
\hline [O III $]$ & 5008.24 & $10017.76^{3)}$ & 850 & 1.0003 & 79.5 \\
\hline
\end{tabular}

NoTes:

1) Heliocentric rest frame.

${ }^{2)} \mathrm{C}$ III] fitted with two velocity components.

3) Asymmetric line: wavelength of emission line peak given.

4) Blending with $\mathrm{H} \epsilon$ ?

5) Echelle order gap in the red line-wing possibly causes a lower measured redshift.

of the $\mathrm{C}$ III] and $\mathrm{Mg}$ II emission lines differ by more than a factor of two from the values found by Steidel \& Sargent (1991). We measured a ratio of $W(\mathrm{C} \mathrm{III]}) / W(\mathrm{Mg} I \mathrm{I})=1.48$, which differs from the ratio $W(\mathrm{C}$ III $]) / W(\mathrm{Mg}$ II $)=0.65$ seen by Steidel \& Sargent (1991). This difference comes mainly from a much smaller $\mathrm{Mg}$ II equivalent width in our spectra. Steidel \& Sargent (1991) see a narrow Mg II component with $\Delta V_{F W H M}^{\mathrm{obs}}=6000 \mathrm{~km} \mathrm{~s}^{-1}$ and a broad component with $\Delta V_{F W H M}^{\mathrm{obs}}=22400 \mathrm{~km} \mathrm{~s}^{-1}$, with the broad component dominating the total $\mathrm{Mg}$ II equivalent width. In our spectra, $\mathrm{Mg}$ II is well fitted by a single Gaussian component of similar equivalent width but a factor of 2 smaller FWHM compared to the narrow component seen by Steidel \& Sargent (1991). We do not see any sign of a broad $\mathrm{Mg}$ II component. Note that $\mathrm{C}$ III] can be decomposed into a narrow and a broad component (see Table 2). Here, the overall FWHM is similar to the value observed by Steidel \& Sargent (1991), but the equivalent width is smaller by a factor of 1.5 . We do not see any signs of blending with Si III] 1892, and Al III 1857 would lie outside our fitted $\mathrm{C}_{\mathrm{III}}$ ] line if appearing at similar redshift. The observed differences might be caused by the optical variability of PKS 2145+067, i.e. the intrinsic Baldwin effect (Baldwin 1977; Pogge \& Peterson 1992; Osmer \& Shields 1999), but unfortunately we do not have a reliable flux calibration for our spectra to quantify this effect (cf. Sect. 2.2).

Here, we focus on the intervening $\mathrm{Mg}$ II absorption system at $z_{\mathrm{abs}}=0.79089$. No other absorption systems toward PKS $2145+067$ could be confirmed by our data. We will present the Galactic absorption lines, redshifted absorption systems, and quasar emission lines for the complete sample of

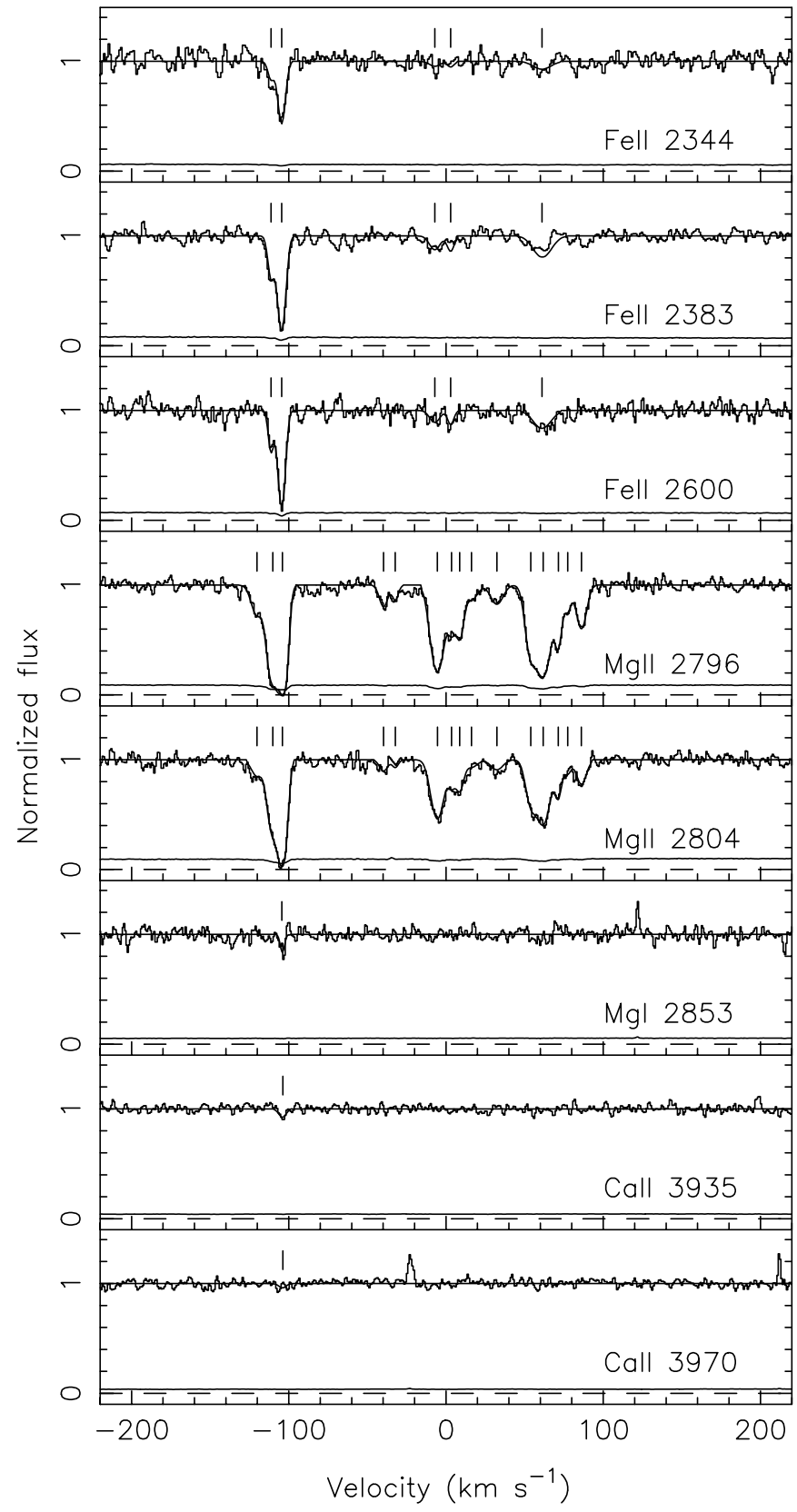

Fig. 1. UVES data for detected absorption lines at $z_{\mathrm{abs}}=0.79089$ in the spectrum of PKS $2145+067$. The velocity scale is for the restframe relative to the absorption redshift. Spectra are superposed with the fitted overall line profile and labelled with the ion and the vacuum transition wavelength. Ticks above the spectra stand for the Voigt profile centroids of each fitted velocity component. The 1-sigma error spectra appear close to the dashed zero level.

quasars in two companion papers (Tappe \& Black 2004, in preparation).

\subsection{Intervening absorption: The $z_{a b s}=0.79089$ system}

Figure 1 shows the UVES data for the detected absorption lines. Spectra are plotted in histogram style superposed with the fitted line profile. The 1-sigma error spectra appear close to the 
Table 3. The $z_{\text {abs }}=0.79089$ absorption system in PKS $2145+067$.

\begin{tabular}{|c|c|c|c|c|c|c|c|}
\hline Ion & $\begin{array}{c}\lambda_{\text {vac, rest }} \\
(\AA)\end{array}$ & $\begin{array}{c}\text { Velocity } \\
\text { component }^{\text {a) }}\end{array}$ & $z_{\text {line }}$ & $\begin{array}{l}\text { Velocity }{ }^{\mathrm{b})} \\
\left(\mathrm{km} \mathrm{s}^{-1}\right)\end{array}$ & $\begin{array}{l}\log N \\
\left(\mathrm{~cm}^{-2}\right)\end{array}$ & $\begin{array}{l}\sum \log N \\
\left(\mathrm{~cm}^{-2}\right)\end{array}$ & $\begin{array}{c}b \\
\left(\mathrm{~km} \mathrm{~s}^{-1}\right)\end{array}$ \\
\hline \multirow[t]{15}{*}{$\mathrm{Mg}_{\text {II }}$} & $2796.3543 /$ & 1 & 0.79017 & $-120.1 \pm 1.5$ & $11.789 \pm 0.168$ & & $4.48 \pm 1.35$ \\
\hline & $2803.5315^{\mathrm{c})}$ & 2 & 0.79023 & $-110.1 \pm 0.7$ & $12.589 \pm 0.136$ & $13.170 \pm 0.095$ & $3.90 \pm 0.82$ \\
\hline & & $3^{\mathrm{d})}$ & 0.79027 & $-104.0 \pm 0.3$ & $13.012 \pm 0.075$ & & $2.73 \pm 0.33$ \\
\hline & & 4 & 0.79065 & $-39.6 \pm 0.8$ & $11.495 \pm 0.125$ & & $3.13 \pm 1.30$ \\
\hline & & 5 & 0.79070 & $-32.2 \pm 1.2$ & $11.192 \pm 0.215$ & & $1.98 \pm 2.63$ \\
\hline & & 6 & 0.79086 & $-5.4 \pm 0.3$ & $12.510 \pm 0.029$ & & $5.00 \pm 0.33$ \\
\hline & & 7 & 0.79091 & $3.6 \pm 2.2$ & $11.762 \pm 0.597$ & $12.769 \pm 0.184$ & $2.40 \pm 1.70$ \\
\hline & & 8 & 0.79094 & $8.8 \pm 1.7$ & $11.947 \pm 0.427$ & & $2.92 \pm 3.43$ \\
\hline & & 9 & 0.79099 & $16.3 \pm 5.0$ & $11.471 \pm 0.525$ & & $4.90 \pm 7.35$ \\
\hline & & 10 & 0.79108 & $32.4 \pm 0.8$ & $11.607 \pm 0.084$ & & $5.05 \pm 1.49$ \\
\hline & & 11 & 0.79121 & $54.0 \pm 1.2$ & $12.227 \pm 0.232$ & & $4.04 \pm 0.54$ \\
\hline & & 12 & 0.79126 & $61.9 \pm 0.8$ & $12.591 \pm 0.106$ & & $5.30 \pm 1.57$ \\
\hline & & 13 & 0.79132 & $71.6 \pm 0.7$ & $11.980 \pm 0.195$ & $12.887 \pm 0.149$ & $2.40 \pm 1.30$ \\
\hline & & 14 & 0.79135 & $77.6 \pm 1.8$ & $11.491 \pm 0.359$ & & $2.34 \pm 3.83$ \\
\hline & & 15 & 0.79141 & $86.3 \pm 0.5$ & $11.931 \pm 0.051$ & & $3.87 \pm 0.83$ \\
\hline $\mathrm{Mg}_{\mathrm{I}}$ & 2852.9631 & 3 & 0.79027 & $-103.9 \pm 0.7$ & $10.705 \pm 0.139$ & & $1.02 \pm 1.65$ \\
\hline \multirow[t]{6}{*}{$\mathrm{Fe}_{\text {II }}$} & $2344.2139 /$ & 2 & 0.79023 & $-111.2 \pm 0.5$ & $12.109 \pm 0.106$ & & $1.57 \pm 0.76$ \\
\hline & $2382.7652 /$ & 3 & 0.79027 & $-104.5 \pm 0.2$ & $12.856 \pm 0.042$ & $12.927 \pm 0.051$ & $2.23 \pm 0.34$ \\
\hline & $2600.1729^{\mathrm{e})}$ & & & & & & \\
\hline & & 6 & 0.79085 & $-7.1 \pm 1.5$ & $11.744 \pm 0.180$ & & $4.14 \pm 2.65$ \\
\hline & & 7 & 0.79091 & $3.1 \pm 1.2$ & $11.612 \pm 0.200$ & $11.984 \pm 0.188$ & $2.08 \pm 2.51$ \\
\hline & & 12 & 0.79126 & $61.2 \pm 1.2$ & $12.166 \pm 0.081$ & & $7.47 \pm 1.85$ \\
\hline $\mathrm{Fe}_{\mathrm{I}}{ }^{\mathrm{f}}$ & & & & & $<11.211$ & & \\
\hline Ca II & $\begin{array}{l}3934.7750 / \\
3969.5901^{\mathrm{c})}\end{array}$ & 3 & 0.79027 & $-103.9 \pm 0.7$ & $10.849 \pm 0.105$ & & $2.81 \pm 1.26$ \\
\hline
\end{tabular}

Notes:

a) The velocity component number is given with respect to $\mathrm{Mg}$ II for all ions; Subsystems are separated by blank lines.

b) Non-relativistic Doppler velocity relative to $z_{\mathrm{abs}}=0.79089$.

c) Simultaneous fit of both doublet components.

d) Saturated velocity component.

e) Simultaneous fit of three Fe II transitions; Fe II 2374 was not visible due to an artifact in the spectrum;

$\mathrm{Fe}$ II 2587 was detected but not included in the analysis because of poor resolution.

f) The upper limit was derived from a combined spectrum of eleven different Fe I transitions (cf. Sect. 2.2 and Fig. 2).

dashed zero level. Ticks above the spectra stand for the Voigt profile centroids of each fitted velocity component. The velocity scale is for the rest-frame relative to the absorption redshift $z_{\text {abs }}=0.79089$. We defined the absorption redshift as the average redshift of all velocity component pairs of the $\mathrm{Mg}$ II doublet (see Table 3).

The $z_{\mathrm{abs}}=0.79089$ system in PKS $2145+067$ shows a rich $\mathrm{Mg}$ II absorption complex with 15 fitted velocity components spanning a range of about $206 \mathrm{~km} \mathrm{~s}^{-1}$. It shows three groups of absorption lines with 3, 7, and 5 components respectively, at velocities of about -130 to $-95 \mathrm{~km} \mathrm{~s}^{-1},-43$ to $41 \mathrm{~km} \mathrm{~s}^{-1}$, and 45 to $93 \mathrm{~km} \mathrm{~s}^{-1}$. Note that "group" is not defined rigorously here; groups are simply separated by one or more resolution elements with a continuum flux consistent to one. Ten of the separations between adjacent features are $10 \mathrm{~km} \mathrm{~s}^{-1}$ or less. Moreover, all of the derived Doppler parameters are less than $6 \mathrm{~km} \mathrm{~s}^{-1}$. Thus it is evident that spectra with resolution coarser than $10 \mathrm{~km} \mathrm{~s}^{-1}$ are incapable of providing an adequate description of this $\mathrm{Mg}$ II absorption system.

The velocity structure of the strongest Mg II components appears also in several Fe II transitions. In addition, we detected weak features of $\mathrm{Mg}$ I and $\mathrm{Ca}$ II at the velocity of the strongest $\mathrm{Mg}$ II component at $-104 \mathrm{~km} \mathrm{~s}^{-1}$. We list the measured line properties in Table 3 . 


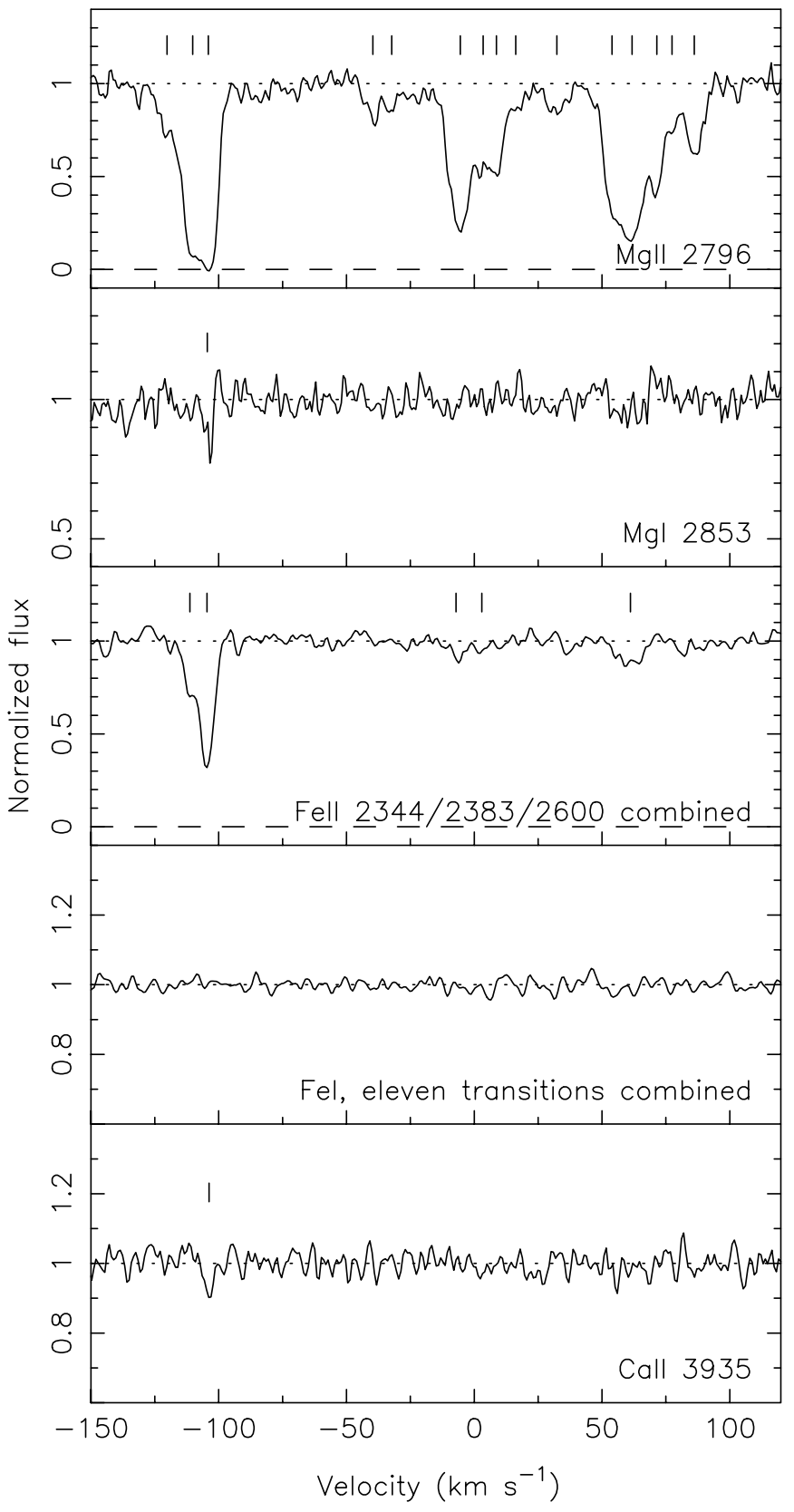

Fig. 2. Detailed view of absorption lines at $z_{\mathrm{abs}}=0.79089$ in the spectrum of PKS $2145+067$. The velocity scale is for the rest-frame relative to the absorption redshift. The normalized continuum level is indicated by a dotted line. Ticks above the spectra stand for the Voigt profile centroids of each fitted velocity component. There is no apparent feature in the combined spectrum of $\mathrm{Fe}$. Note also that the plotted flux ranges differ for the individual transitions in order to enhance the visibility of weak features.

\section{Discussion of the absorbing system}

\subsection{Kinematics}

The ultimate aim of studying quasar absorption line systems at various redshifts is to understand the structure and evolution of the absorbing clouds and their associated galaxies. The measured velocities, line-widths, and column densities can be interpreted in terms of a model of the absorbing system. Primarily, it is the nature of the absorbing clouds, their macroscopic and internal motions as well as their chemical and physical conditions that determine the observed line characteristics. In turn, the cloud properties are connected to their spatial positions within the galaxy through the stellar and extragalactic background radiation field (ionization conditions) and the overall galaxy structure and kinematics.

Here, we consider the case of a galaxy at a certain cosmological redshift, $z_{\mathrm{cos}}$, absorbing light from a continuum background source. The observed redshifts inferred from the wavelengths of the absorption lines, $z_{\text {line, }}$, are the combination of the cosmological redshift due to the expansion of space in an expanding universe and the Doppler shift due to the physical motion of the absorbing gas. The physical motions are commonly called kinematics of the absorbing system, but it is actually not clear how to separate them from the cosmological redshift. Physical motions in this context often denote large-scale motions dominated by gravity, for example orbital motions of galaxies in a galaxy cluster, but they also include the gas dynamics of the absorbing gas. The redshift due to physical motion relative to a chosen standard of rest can be described by the special-relativistic Doppler formula for a locally flat spacetime.

In practice, since the distance to the galaxy is unknown a priori, the cosmological redshift must be fixed by definition, for example through averaging the redshifts of all observed absorption lines. Even when the true cosmological redshift is known, it is not clear how to distinguish the local gas motions from the motion of the galaxy as a whole. However, the velocity differences between the individual velocity components stay the same regardless of the cosmological redshift and the galaxy motion. More accurately, the observed velocity spread is a lower limit of the true velocity spread in the absorbing system, because the observed relativistic Doppler shift is strongly dependent on the angle at which we see the moving source. Without independent information about transverse motion, we measure only the radial component of motion; therefore, the range of speeds in the absorbing system may be much higher.

The observed kinematics show a dominant, saturated group of absorption lines accompanied by weaker groups of larger kinematic spread. The first group of absorption lines contains about 50 percent of the total $\mathrm{Mg}$ II column density and has the smallest velocity spread of about $16 \mathrm{~km} \mathrm{~s}^{-1}$. Furthermore, it is notable that all fitted components for all detected ions agree in velocity within the error limits (about $1 \mathrm{~km} \mathrm{~s}^{-1}$ ), although no constraints were used for the fitting. Since the ions have different ionization potentials lying above ( $\mathrm{Mg}$ II, Fe II) and below (Mg I, Fe I, Ca II) the hydrogen edge of $13.6 \mathrm{eV}$, their lines would appear at different velocities if the clouds had an internal velocity and ionization structure. Stratified ionization structure would normally require that the cloud be optically thick in the $\mathrm{H}$ continuum, i.e. $N(\mathrm{HI}) \geq 2 \times 10^{17} \mathrm{~cm}^{-2}$.

Although we do not attempt to produce a detailed model of the kinematics for PKS 2145+067, we find it difficult to explain the large observed kinematic spread of the $\mathrm{Mg}_{\text {II }}$ absorption complex. For PKS $2145+067$, the projected impact parameter of the quasar sight line to the absorbing galaxy derived from the observed angular separation between the identified absorber galaxy and the quasar on the sky is $29 h^{-1} \mathrm{kpc}$ 
(Bergeron \& Boissé 1991, cf. Sect. 4.2, Eq. (7)). A simple model with a corotating, spherically symmetric halo (geometry described by Weisheit 1978) with an assumed radius of $180 h^{-1} \mathrm{kpc}$ (cf. Chen et al. 2001) can only reproduce the observed spread of $206 \mathrm{~km} \mathrm{~s}^{-1}$ by assuming large constant rotation velocities $\gtrsim 300 \mathrm{~km} \mathrm{~s}^{-1}$ and/or small inclination angles. For a more realistic kinematic model of the absorber geometry, high spatial resolution imaging is needed to measure the rotation curve and the orientation of the absorber host galaxy (cf. Steidel et al. 2002).

\subsubsection{Line broadening}

The line-widths or $b$-parameters provide an upper limit on the kinetic gas temperature (cf. Sect. 2.3.1). We derive $T \leq 25600 \mathrm{~K}$ from an average $\langle b\rangle \leq 4.2 \mathrm{~km} \mathrm{~s}^{-1}$ for all fitted $\mathrm{Mg}$ II components with fractional errors smaller than unity. The strongest component of $\mathrm{Mg}$ II has the narrowest well measured line width, $b=2.73 \pm 0.33$, which corresponds to $T \leq 13500 \mathrm{~K}$. $\mathrm{Mg}_{\text {I }}$ has a smaller $b$-parameter, $b=1.02 \pm 1.65$, although the error is large. The upper temperature limit derived from $\mathrm{Mg}$ I is $T \leq 6500 \mathrm{~K}$, or if the maximum error is added to the measured $b$-parameter, $T \leq 10500 \mathrm{~K}$.

We also tested for turbulent broadening by comparing the ratio of $b$-parameters, $b_{\mathrm{Mg} \text { II }} / b_{\mathrm{Fe} \text { II }}$, with the inverse ratio of the square root of the ion masses (cf. Sect. 2.3.1, Eq. (4)). For example, the ratio $b_{\mathrm{MgI}(2)} / b_{\mathrm{Fe} \mathrm{II}(2)}=2.48 \pm 1.31$ for velocity component 2 is consistent with the thermal ratio $(24.305 / 55.847)^{-0.5}=1.52$, i.e. consistent with no turbulent broadening. Checking the ratios of components 3,6 , and 7 , and doing the same calculation with the ratio $b_{\mathrm{Mg} \text { II }} / b_{\mathrm{Ca} \text { II }}$ for component 3 confirmed the previous result. The ratio $b_{\mathrm{MgII}(3)} / b_{\mathrm{Fe} \mathrm{II}(3)}=1.22 \pm 0.24$ is marginally inconsistent with the thermal ratio, but this inconsistency is removed by assuming an error slightly larger than the formal value for the $b$-parameter of the strongly saturated $\mathrm{Mg}$ II(3) component.

\subsection{Abundances and ionization state}

The detection of both $\mathrm{Mg}$ II and Mg I suggests the study of the ionization state of the absorber for the velocity component where the absorption arises. Although additional atoms and ions including $\mathrm{H}_{\mathrm{I}}, \mathrm{C}_{\mathrm{IV}}, \mathrm{Si}$ IV, and $\mathrm{O}$ VI, have been observed in low-resolution HST/FOS spectra, their abundances cannot be determined with high accuracy (Bergeron et al. 1994). Other published ionization analyses have been applied statistically to samples of $\mathrm{Mg}$ II absorbers (Churchill et al. 2003); as a result, some interesting details of the analysis are obscured.

We developed a compact photoionization model to evaluate the rates of processes that govern the ionization balance of magnesium. Our model takes a given radiation field and an observed value of $N\left(\mathrm{Mg}^{+}\right) / N(\mathrm{H})$, and returns the value of the $N\left(\mathrm{Mg}^{+}\right) / N(\mathrm{Mg})$ ratio for a grid of electron temperatures $T_{\mathrm{e}}$ and total hydrogen densities $n_{\mathrm{H}}$. It particularly takes into account charge transfer (Allan et al. 1988), dielectronic recombination (Nussbaumer \& Storey 1986), and electron impact ionization (Lotz 1967). The classical-empirical formula of Lotz (1967) agrees within a factor of two with the rates derived from recent measurements of the Mg electron-impact ionization cross sections (Boivin \& Srivastava 1998). Rate coefficients of radiative recombination have been re-computed to incorporate new cross sections of photoionization from low-lying states of neutral Mg (Mendoza \& Zeippen 1987).

In particular, we consider the constraints provided by the $\mathrm{Mg}$ II and $\mathrm{Mg}$ I lines and the neutral hydrogen column density from a reanalysis of archived low-resolution HST/FOS spectra. From our high-resolution data, we derive a ratio of column densities in component 3 of $N\left(\mathrm{Mg}^{+}\right) / N(\mathrm{Mg})=203 \pm 74$. Our analysis of the HST/FOS spectra using the observed velocity distribution of $\mathrm{Mg}_{\text {II }}$ yields an upper limit for the total neutral hydrogen column density of $N(\mathrm{H}) \leq 1 \times 10^{18} \mathrm{~cm}^{-2}$, which is consistent with a previous analysis by Bergeron et al. (1994). If the neutral hydrogen is indeed distributed in velocity like the observed $\mathrm{Mg}^{+}$, then the column density of $\mathrm{H}$ in component 3 would be $N(\mathrm{H}) \approx 3.6 \times 10^{17} \mathrm{~cm}^{-2}$. This column density is low enough that the optical depth to ionizing photons at the Lyman limit is only of the order of unity, and we can treat the ionization of both magnesium and hydrogen in the optically thin limit to a good approximation.

The shape and intensity of the radiation field at the physical object is one of the major uncertainties in photoionization calculations. In the case of PKS 2145+067, Bergeron \& Boissé (1991) have identified the galaxy associated with the $\mathrm{Mg}$ II absorber, and have obtained a low-resolution spectrum in the range 4100-7000 $\AA$. The galaxy is bright, with $M_{\mathrm{B}} \approx-20.35$ ( $h=0.71$ ), corresponding to $L / L_{*} \approx 1$ for $M_{\mathrm{B}, *}=-20.24$ (Loveday et al. 1992). It shows signs of star-formation, based on [O II] 3727 emission with an equivalent width $W_{\text {obs }}=28 \AA$.

We fitted a power-law with slope 0.8 to the observed spectrum, and estimated a flux density of $J_{\lambda}^{z=0}(1633 \AA) \approx 1 \times$ $10^{-18} \mathrm{erg} \mathrm{s}^{-1} \mathrm{~cm}^{-2} \AA^{-1}$ at $1633 \AA$ by extrapolating the fit and taking into account Galactic extinction. The Lyman limit flux density at the position of the absorber, $J_{\lambda}^{z_{\text {abs }}=0.79}(912 \AA)$, can then be calculated as follows:

$J_{\lambda}^{\text {zabs }=0.79}(912 \AA)=\frac{L_{\lambda}(912 \AA)}{4 \pi r_{\text {abs }}^{2}}$,

with

$L_{\lambda}(912 \AA)=4 \pi D_{\mathrm{L}}^{2}\left(1+z_{\mathrm{abs}}\right) J_{\lambda}^{z=0}(1633 \AA)$,

and

$r_{\mathrm{abs}}=D_{\mathrm{A}} \theta_{\mathrm{abs}}$,

where $L_{\lambda}(912 \AA)$ is the flux of the galaxy at the Lyman limit, $z_{\mathrm{abs}}$ is the redshift of the absorber and the galaxy, $D_{\mathrm{L}}$ and $D_{\mathrm{A}}$ are the cosmological luminosity and angular distances, $r_{\mathrm{abs}}$ is the distance from the absorber to the galaxy, and $\theta_{\text {abs }}$ is the observed angular separation between the galaxy and the absorber on the sky in radians. Note that we used $r_{\text {abs }}$ instead of $D_{\mathrm{L}}$ in Eq. (5) to excellent approximation because the distance between absorber and galaxy is very small in terms of redshift. We calculated $D_{\mathrm{A}}=1544.6 \mathrm{Mpc}$ with the routines provided by Kayser et al. (1997) for a given cosmological 
Table 4. Power-law radiation field parameters, $J_{v}=J_{v_{0}}\left(v / v_{0}\right)^{\beta}$, used for the photoionization model of the $z=0.79 \mathrm{Mg}$ II absorber toward PKS $2145+067$.

\begin{tabular}{ccc}
\hline \hline $\begin{array}{c}\text { Wavelength range, } \lambda_{0}-\lambda \\
(\AA)\end{array}$ & $\begin{array}{c}\text { Flux density } J_{v_{0}} \\
\left(\mathrm{erg} \mathrm{s}^{-1} \mathrm{~cm}^{-2} \mathrm{~Hz}^{-1} \mathrm{sr}^{-1}\right)\end{array}$ & Slope $\beta$ \\
\hline $90-200$ & $2.09 \times 10^{-24}$ & -0.17 \\
$200-400$ & $2.37 \times 10^{-24}$ & -4.54 \\
$400-912$ & $5.93 \times 10^{-23}$ & -1.21 \\
$912-1621$ & $6.54 \times 10^{-22}$ & -2.89 \\
\hline
\end{tabular}

model $\left(\Omega_{\Lambda}=0.73, \Omega_{\mathrm{m}}=0.27, h=0.71\right) . D_{\mathrm{L}}$ is simply related to $D_{\mathrm{A}}$ by $D_{\mathrm{L}}=(1+z)^{2} D_{\mathrm{A}}$.

Using Eqs. (5)-(7) and the observed angular separation of 5'.5 (Bergeron \& Boissé 1991), we estimate a Lyman limit flux density at the $\mathrm{Mg}$ II absorber of $J_{\lambda}^{\text {zabs }=0.79}(912 \AA) \approx$ $2.6 \times 10^{-8} \mathrm{erg} \mathrm{s}^{-1} \mathrm{~cm}^{-2} \AA^{-1}$, which corresponds to $5.7 \times$ $10^{-22} \mathrm{erg} \mathrm{s}^{-1} \mathrm{~cm}^{-2} \mathrm{~Hz}^{-1} \mathrm{sr}^{-1}$ in frequency units per steradian. This value is prone to some uncertainty due to the extrapolation, however, it is about a factor of five larger than the estimated contribution to the UV background from all star-forming galaxies at $z \approx 0.5$ and with an escape fraction of 1 (Scott et al. 2002). On the other hand, it is about five times smaller than the flux of a typical $z \approx 0.01$ starburst galaxy (Buat et al. 2002) placed at the same distance to the absorber.

Haardt \& Madau (1996) calculated spectra of the UV background as a function of redshift due to QSO emission alone. We estimate a flux density of $J_{v}^{z=0.79}(912 \AA) \approx 1.1 \times$ $10^{-22} \mathrm{erg} \mathrm{s}^{-1} \mathrm{~cm}^{-2} \mathrm{~Hz}^{-1} \mathrm{sr}^{-1}$ at $z=0.79$ at the hydrogen Lyman limit by interpolating their data given for redshifts of $0,0.5$, 1 , and 1.5, with a low-order polynomial. Again, this value is about a factor of five smaller than the estimated flux from the galaxy associated with the $z=0.79 \mathrm{Mg}$ II absorber toward PKS 2145+067. Hence, the photoionization of that absorber, in particular the photoionization of neutral magnesium at wavelengths smaller than $1621 \AA$, is probably dominated by the UV flux from the associated galaxy.

We adopt a simple composite power-law model for the radiation field in our photoionization model (see Table 4). In the important wavelength range of 912-1621 (Ly limit - Mg first ionization potential), we take the slope and intensity from the spectrum of the local galaxy derived above plus the contribution from the Haardt \& Madau (1996) pure quasar background at $z=0.79$. Below the Lyman limit, we adopt the Haardt \& Madau (1996) spectrum approximated by a joined set of power laws together with a 10 percent contribution from the local galaxy, i.e. we assumed a 10 percent escape fraction below the Lyman limit. The fine spectral details of the original Haardt \& Madau (1996) data that are lost due to the power-law approximation are only of minor importance for our photoionization model.

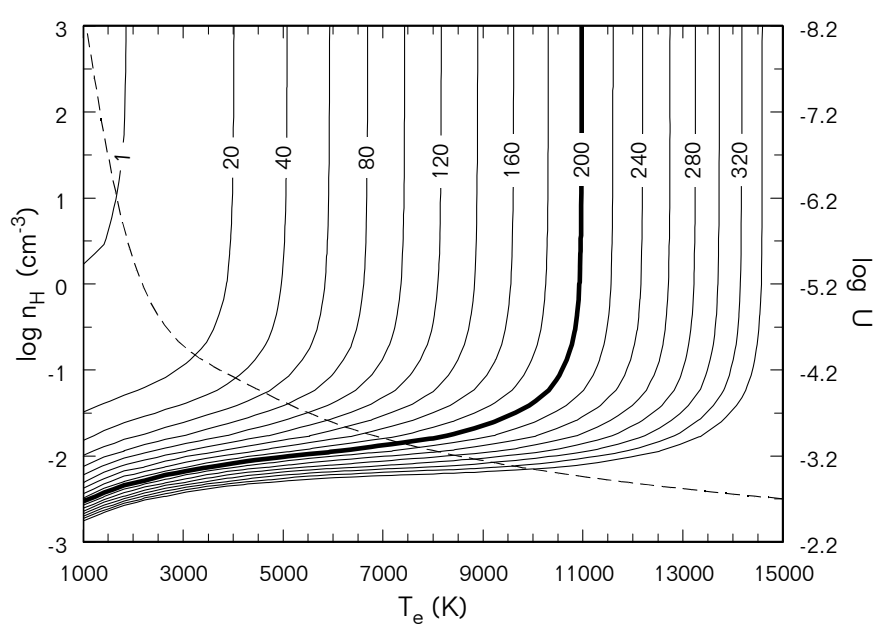

Fig. 3. Photoionization model results for the $z=0.79 \mathrm{Mg}$ II absorber toward PKS 2145+067. The solid lines depict the ratio of $\mathrm{Mg}^{+} / \mathrm{Mg}$ in the range 1 to 340 for a grid of electron temperatures and total hydrogen densities/ionization parameters. Lines for $\mathrm{Mg}^{+} / \mathrm{Mg}$ ratios larger than 340 are not displayed. The thick solid line corresponds to the observed $\mathrm{Mg}^{+} / \mathrm{Mg}$ column density ratio (cf. Sect. 4.2). The dashed line represents grid points where the rate of $\mathrm{Mg}+\mathrm{H}^{+}$charge transfer equals the rate of photoionization (cf. Figs. 4 and 5). Above the dashed line, the collisional charge-transfer process rather than photoionization becomes the dominating source of ionization of $\mathrm{Mg}$. See Sect. 4.2.1 for further discussion.

\subsubsection{Photoionization model results}

The ratio of $\mathrm{Mg}^{+} / \mathrm{Mg}$ densities in steady-state is given by:

$\frac{n\left(\mathrm{Mg}^{+}\right)}{n(\mathrm{Mg})}=\frac{\gamma_{\mathrm{Mg}}+n_{\mathrm{e}}\left(q_{\mathrm{Mg}+\mathrm{e}}+q_{\mathrm{Mg}+\mathrm{H}^{+}}\right)}{n_{\mathrm{e}} \alpha_{\mathrm{Mg}^{+}+\mathrm{e}}}$,

where $\gamma_{\mathrm{Mg}}$ is the photoionization rate of $\mathrm{Mg}, n_{\mathrm{e}}$ is the electron density, $q_{\mathrm{Mg}+\mathrm{e}}$ is the rate coefficient of $\mathrm{Mg}$ electron impact ionization, $q_{\mathrm{Mg}+\mathrm{H}^{+}}$is the rate coefficient of $\mathrm{Mg}+\mathrm{H}^{+}$charge transfer, and $\alpha_{\mathrm{Mg}^{+}+\mathrm{e}}$ is the total dielectronic and radiative rate coefficient of $\mathrm{Mg}^{+}$recombination. We ignored the reverse charge transfer process $\mathrm{Mg}^{+}+\mathrm{H}$, which is too slow to compete with recombination in the conditions of interest.

We present the results from our photoionization model for the $z=0.79 \mathrm{Mg}$ II absorber toward PKS 2145+067 in Fig. 3. The solid lines represent the ratio of $\mathrm{Mg}^{+} / \mathrm{Mg}$ for a grid of electron temperatures and total hydrogen densities, with the thick line representing the observed $\mathrm{Mg}^{+} / \mathrm{Mg}$ column density ratio. Our model shows that there are basically two types of solutions: a low-density solution with densities smaller than about $0.05 \mathrm{~cm}^{-3}$ and temperatures in the range of $1000-10000 \mathrm{~K}$, and a high-density solution where the temperature is nearly constant at $\approx 11000 \mathrm{~K}$ and the density is free to vary between 0.1 and $1000 \mathrm{~cm}^{-3}$.

The $\mathrm{Mg}^{+} / \mathrm{Mg}$ ratio in Fig. 3 is governed by the rates in Eq. (8), which are displayed in Figs. 4-5 for a low- and a high-density case. It is illustrative to look at these details of atomic physics to gain a deeper understanding of the processes at work in the $\mathrm{Mg}$ II absorber. Since the hydrogen is partly ionized by the extragalactic background radiation, most of the free 


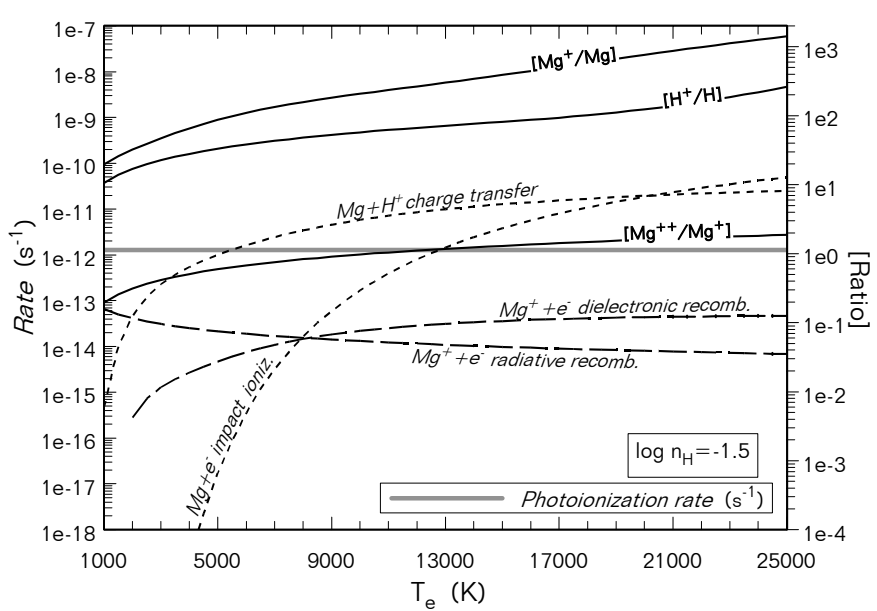

Fig. 4. Rates that govern the Mg ionization balance in our photoionization model as functions of electron temperature for a constant density of $\log n_{\mathrm{H}}=-1.5 \mathrm{~cm}^{-3}$. Rates of collisional processes, i.e. products of rate coefficients and electron density, are depicted as dashed lines, the thick solid grey line is the constant photoionization rate, and ionic/atomic ratios are given as solid lines. See Sect. 4.2.1 for a discussion.

electrons probably come from ionization of $\mathrm{H}$. As long as the temperature of the absorbing gas exceeds a few thousand $\mathrm{K}$, the rate of charge-transfer, $\mathrm{H}^{+}+\mathrm{Mg} \rightarrow \mathrm{H}+\mathrm{Mg}^{+}$, is high enough (Allan et al. 1988) that this process may be a more important source of $\mathrm{Mg}^{+}$than photoionization of $\mathrm{Mg}$ in the extragalactic background. In that case, the $\mathrm{Mg}^{+} / \mathrm{Mg}$ ratio is mainly a function of temperature when the total hydrogen density exceeds $0.1 \mathrm{~cm}^{-3}$ (see Fig. 3). The dashed line in the figure marks the points where the charge transfer rate, that is the product of rate coefficient and electron density, becomes equal to the photoionization rate. Note that the temperature-dependence of the total recombination rate is similar to that of charge transfer at temperatures above $\approx 5000 \mathrm{~K}$, and thus the $\mathrm{Mg}^{+} / \mathrm{Mg}$ ratio remains constant when controlled solely by collisional processes.

Two changes become evident when comparing the lowdensity case in Fig. 4 with the high-density case in Fig. 5. For higher densities, all collisional processes gain importance relative to the constant photoionization rate due to the higher electron density. Thus the $\mathrm{Mg}^{+} / \mathrm{Mg}$ ratio drops drastically at low temperatures compared to the low-density case due to the dominance of radiative recombination. At higher temperatures the $\mathrm{Mg}^{+} / \mathrm{Mg}$ ratios are similar in both density cases, which is the collision dominated situation described above. Furthermore, it is interesting to note the change at temperatures of about $13000 \mathrm{~K}$, where the electron impact ionization of atomic hydrogen becomes an important source of electrons. However, this leaves the $\mathrm{Mg}^{+} / \mathrm{Mg}$ ratio unaffected since the increase in electron density affects recombination and charge transfer/electron impact ionization alike (cf. Eq. (8)). In contrast, the $\mathrm{Mg}^{++} / \mathrm{Mg}^{+}$ratio is affected, since photoionization is the dominant source of $\mathrm{Mg}^{++}$. The increasing electron density causes a higher radiative recombination rate, which decreases the $\mathrm{Mg}^{++} / \mathrm{Mg}^{+}$ratio (note also that dielectronic recombination is not of importance for $\mathrm{Mg}^{++}$).

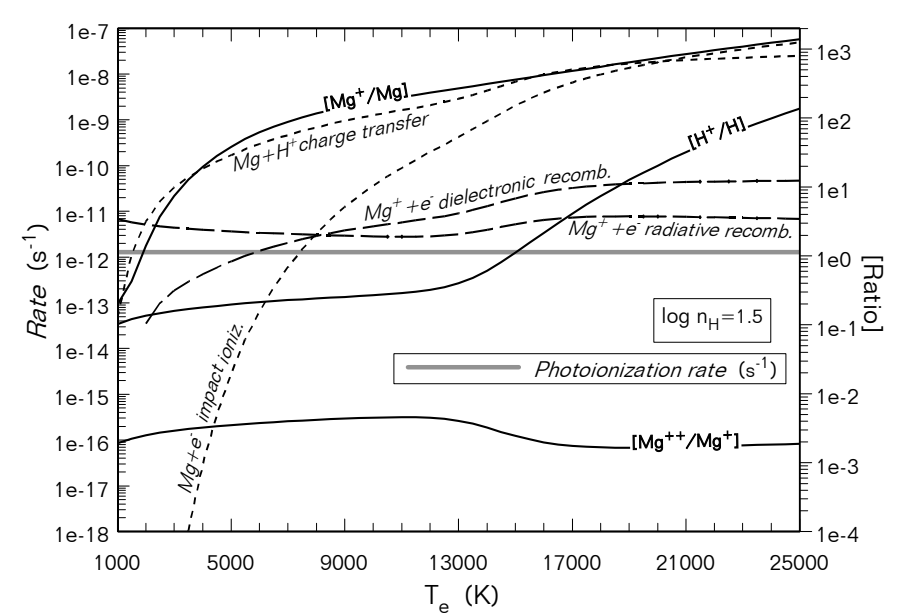

Fig. 5. Rates that govern the Mg ionization balance in our photoionization model as functions of electron temperature for a constant density of $\log n_{\mathrm{H}}=1.5 \mathrm{~cm}^{-3}$. Rates of collisional processes, i.e. products of rate coefficients and electron density, are depicted as dashed lines, the thick solid grey line is the constant photoionization rate, and ionic/atomic ratios are given as solid lines. See Sect. 4.2.1 for a discussion.

Finally, what conclusions can be drawn regarding the situation for the $z=0.79 \mathrm{Mg}$ II absorber toward PKS 2145+067? From Fig. 3 it becomes clear that a constraint on the temperature and/or the total hydrogen density is needed to give a unique solution. This is generally hard to achieve for $\mathrm{Mg}$ II absorbers, but we can put loose bounds on the temperature by the following arguments.

First, we can use a constraint on the $\mathrm{Mg}$ abundance in the absorber. The abundance constraint allows us to obtain a lower limit on the cloud size, $L_{\min }$, from Eq. (9):

$L_{\min }=\frac{N(\mathrm{Mg})+N\left(\mathrm{Mg}^{+}\right)\left[\left[\mathrm{Mg}^{++} / \mathrm{Mg}^{+}\right]+1\right]}{[\mathrm{Mg} / \mathrm{H}]_{\max } n_{\mathrm{H}}}$.

We can calculate the size $L$ of the absorber from the model results and the measured total $\mathrm{H}$ column density, with the assumption that the absorber is uniform and the neutral $\mathrm{H}$ is distributed in velocity like $\mathrm{Mg}$ II. The size then becomes $L=N(\mathrm{H})\left(1+\left[\mathrm{H}^{+} / \mathrm{H}\right]\right) / n_{\mathrm{H}}$, which ranges from about $10^{-4} \mathrm{pc}$ at $11000 \mathrm{~K}$ and $1000 \mathrm{~cm}^{-3}$ to $4600 \mathrm{pc}$ at $1000 \mathrm{~K}$ and $0.003 \mathrm{~cm}^{-3}$ (following the thick solid line in Fig. 3). Comparing both derived sizes $L$ and $L_{\min }$ using a Galactic ISM Mg abundance of $[\mathrm{Mg} / \mathrm{H}]_{\mathrm{ISM}}^{\text {Galactic }}=1.1 \times 10^{-6}$, we find that $L$ becomes consistent with $L_{\min }$ for sizes $L \gtrsim 100 \mathrm{pc}$ or temperatures less than about $10000 \mathrm{~K}$.

If the absorber connected to the galaxy at $z=0.79$ is less evolved than present-day galaxies, i.e. having a smaller metallicity than found for the Sun and the Galactic interstellar medium, it is reasonable to adopt a lower abundance: $[\mathrm{Mg} / \mathrm{H}]=0.8 \times 10^{-6}$ would shift the point where $L$ becomes larger than $L_{\text {min }}$ to a lower temperature of about $8500 \mathrm{~K}$ in Fig. 3, corresponding to a cloud size of about $550 \mathrm{pc}$ or larger. It is interesting to note that $L$ is not consistent with $L_{\min }$ in our parameter range for values $[\mathrm{Mg} / \mathrm{H}] \lesssim 0.75 \times 10^{-6}$. In other words, our model predicts a roughly constant abundance of $[\mathrm{Mg} / \mathrm{H}]=0.75 \times 10^{-6}$ for temperatures below $7000 \mathrm{~K}$. 
At those temperatures, cloud sizes start to become uncomfortably large, i.e. larger than $1 \mathrm{kpc}$, which makes it difficult to understand the narrow observed line widths in $\mathrm{Mg}$ II and $\mathrm{Mg}$ I component 3: kinematic velocities should not remain so coherent on such large scales. A magnesium abundance larger than the Galactic value, e.g. $[\mathrm{Mg} / \mathrm{H}]=1 \times 10^{-5}$, would shift the point where $L$ becomes larger than $L_{\min }$ to a higher temperature of about $11000 \mathrm{~K}$ in Fig. 3, corresponding to a cloud size of about $0.2 \mathrm{pc}$ or larger.

Another independent constraint on the temperature comes from the energy balance of a photoionized gas. The temperature at which the gas equilibrates is determined by the intersection of the heating and cooling curves (Ferland 2003). These curves depend on a number of factors, including the incident radiation field, the total abundances of the elements, and the gas-to-dust ratio. The last two factors are difficult to determine for the present case of the $z=0.79 \mathrm{Mg}$ II absorber, but we can make some estimates owing to the fact that photoionized gas tends to equilibrate at distinct temperature regimes. For a lower metallicity $Z / Z_{\text {Sun }}<1$, the equilibrium temperatures tend to be higher due to the less efficient cooling. From hydrogen cooling alone, we estimate an equilibrium temperature of the order of $10^{4} \mathrm{~K}$ for the $z=0.79 \mathrm{Mg}$ II absorber toward PKS $2145+067$. It is hard to put a more stringent upper limit to the temperature since the abundances of metals that serve as efficient coolants are difficult to determine (Sect. 5). High-resolution data of good quality that cover higher ionization species are needed.

Taking all these considerations together, we argue that the temperature is most likely of the order of $10000 \mathrm{~K}$ or below, which implies total hydrogen densities smaller than about $0.04 \mathrm{~cm}^{-3}$ and absorber sizes larger than $100 \mathrm{pc}$ according to our photoionization model.

\subsection{Implications for QSO absorption line studies of a possible fine-structure constant variation}

Recently, evidence for cosmological space/time-variations of the fine-structure constant $\alpha$ has been based on quasar absorption lines from many different multiplets in different ions ("Many-Multiplet method": Dzuba et al. 1999a,b; Murphy et al. 2001a,b, 2003a,b; Webb et al. 2001, 2003). The variation is $\Delta \alpha / \alpha=\left(\alpha_{\mathrm{z}}-\alpha_{0}\right) / \alpha_{0}=(-0.543 \pm 0.116) \times 10^{-5}$ from three independent Keck/HIRES samples with a total of $128 \mathrm{ab}-$ sorption systems over the range $0.2<z_{\text {abs }}<3.7$ (Murphy et al. 2003a). This result stands in contrast to a recent study by Bahcall et al. (2004), who do not see any evidence for a variation in $\alpha$. The latter used strong [O III] 4960/5008 emission lines to put a robust upper limit on the change of $\alpha$, and find $\Delta \alpha / \alpha=(1.2 \pm 0.7) \times 10^{-4}$ from 165 quasars with $z_{\mathrm{em}}<0.8$ selected from the Sloan Digital Sky Survey (SDSS) first data release (DR1, Schneider et al. 2003).

Bahcall et al. (2004) compared the [O III] method and results of previous absorption line studies critically with the Many-Multiplet method, and pointed out the possible influence of velocity structure and undiscovered velocity components. The Many-Multiplet method explicitly assumes that the velocity structure seen in one ion corresponds exactly to that seen in any other ion (Murphy et al. 2001b). Bahcall et al. (2004) point out that differences in relative velocity as small as $0.2 \mathrm{~km} \mathrm{~s}^{-1}$ could give rise to apparent shifts $\Delta \alpha / \alpha$ in individual systems that are of the order of the average $\Delta \alpha / \alpha$ observed with the Many-Multiplet method. They suggest high-resolution absorption-line spectroscopy (resolution $R=10^{5}$ or better) to test the Many-Multiplet assumptions on velocity structure directly. Murphy et al. (2001a, 2003a) argue, however, that these kinematic shifts in $\Delta \alpha / \alpha$ are random, and only increase the scatter of $\Delta \alpha / \alpha$ in a large sample of absorbers. They remark that future analyses of independent spectra from different telescopes and spectrographs will provide a crucial check to the Keck/HIRES results. Such a test has been recently performed with VLT/UVES by Chand et al. (2004) and Quast et al. (2004), who did not find evidence for the variability of $\alpha$. However, Quast et al. have only studied a single Fe II absorption system at a resolution of $R \approx 55000$.

VLT/UVES data of the type presented in this work, with a resolution of $R \approx 10^{5}$, are ideally suited to provide further independent tests of the Keck/HIRES results. The large spectral coverage of VLT/UVES allows the simultaneous measurement of [O III] quasar emission and intervening $\mathrm{Mg}$ II/ $/ \mathrm{Fe}$ II absorption up to a redshift $z=1$, and individual $\mathrm{Mg}_{\text {II }}$ or Fe II absorption up to $z=2.5$. For the individual example of PKS 2145+067, we find that the Many-Multiplet assumption on coinciding velocity structure for all ions is correct within our measurement errors, which are $0.3 \mathrm{~km} \mathrm{~s}^{-1}$ in the best case for the strongest $\mathrm{Mg}$ II and Fe II components (cf. Table 3). Still, we emphasize that Murphy et al. (2003a) have missed about half the velocity components that we find in our data in their analysis for PKS 2145+067. Undiscovered, blended velocity components can be a potential problem for the Many-Multiplet method, especially for the low- $z \mathrm{Mg}$ II/ $/ \mathrm{Fe}_{\text {II }}$ systems in their sample, considering that observed $\mathrm{Mg}$ II absorber kinematics are not random but follow characteristic statistical patterns (cf. Sect. 4.1; Churchill \& Vogt 2001; Churchill et al. 2003). The prevalent observed patterns, i.e. a dominant group of closely spaced absorption lines accompanied by weaker features of larger kinematic spread, are commonly interpreted as a result of the absorber geometry, a rotating disk and/or halo (cf. Sect. 4.1). The velocity structure of individual groups of absorption lines is then determined by the small-scale structure and kinematics of the absorbing material.

A larger number of velocity components detected through high resolution would improve the limit on $\Delta \alpha / \alpha$ determined with the Many-Multiplet method in two ways: (1) reduction of potential systematic errors due to blended, unresolved velocity structure that shows systematic patterns over a large sample; even if the unresolved components are randomly distributed, high-resolution would result in much smaller error bars and a tighter limit on $\Delta \alpha / \alpha$; (2) a larger number of detected components, including improved sensitivity for weak, narrow velocity components, resulting in better statistics. This is most important at redshifts $z \lesssim 1.5$, where most of the measured Mg II absorbers reside, and where cosmological theories with varying $\alpha$ and a positive cosmological constant predict a smaller change of $\alpha$ than at higher redshifts (e.g., Barrow et al. 2002a,b; Sandvik et al. 2002). 


\section{Conclusions}

We obtained a high-resolution, $R \approx 10^{5}$ or $3 \mathrm{~km} \mathrm{~s}^{-1}$, optical spectrum of the quasar PKS 2145+067 with VLT/UVES (Sect. 2.1). Our data analysis (Sect. 2.3) reveals several emission lines from the quasar itself as well as intervening $\mathrm{Mg}$ II absorption at $z_{\mathrm{abs}}=0.79089$. We also detected lines of $\mathrm{Mg}$ I, Fe II, and $\mathrm{Ca}$ II in the same absorber. We refer to a companion paper for the presentation of the Galactic absorption lines (Tappe \& Black 2004).

The observed broad quasar emission lines show substantial differences in $F W H M$ and equivalent width compared to previous measurements by Steidel \& Sargent (1991). This might be interpreted as the intrinsic Baldwin effect (Baldwin 1977; Pogge \& Peterson 1992; Osmer \& Shields 1999) due to the optical variability of PKS $2145+067$ (Sect. 3).

The $z_{\mathrm{abs}}=0.79089$ absorption system shows $15 \mathrm{Mg}$ II velocity components spanning a range of about $206 \mathrm{~km} \mathrm{~s}^{-1}$. We derive slightly higher total column densities for $\mathrm{Mg}_{\text {II }}$ and $\mathrm{Fe}_{\text {II }}$ than Churchill \& Vogt (2001), who found $6 \mathrm{Mg}$ II velocity components in Keck/HIRES spectra of $R \approx 45000$ or $6.6 \mathrm{~km} \mathrm{~s}^{-1}$. Our observations reveal considerably more structure in narrower lines than in the previous work (see Fig. 2 and Table 3). In addition, we detected previously unreported $\mathrm{Mg}$ I and $\mathrm{Ca}$ II at the same velocity as the strongest $\mathrm{Mg}_{\text {II }}$ component, and put a strong upper limit on Fe I (Table 3). High-resolution data of the type presented in this work are ideally suited to provide independent tests of proposed cosmological space/time-variations of the fine-structure constant $\alpha$ (Murphy et al. 2003a): detection of a larger number of velocity components, and improved analysis of blended velocity structure (cf. Sect. 4.3).

We developed a compact photoionization model to evaluate the rates of processes that govern the ionization balance of magnesium, in particular the $\mathrm{Mg}^{+} / \mathrm{Mg}$ ratio (Sect. 4.2.1, Eq. (8)). From our photoionization model, we find the following physical conditions for the $\mathrm{Mg}$ II absorber toward PKS 2145+067: a temperature of the order of $10^{4} \mathrm{~K}$ or below, a total hydrogen density $n_{\mathrm{H}} \lesssim 0.04 \mathrm{~cm}^{-3}$, and an absorber size $L \gtrsim 100 \mathrm{pc}$. This implies a magnesium abundance in the absorber of $7.5 \times 10^{-7} \lesssim[\mathrm{Mg} / \mathrm{H}]_{\mathrm{abs}} \lesssim 10^{-6}$, which is about $0.02-0.03 Z_{\odot}$, taking $[\mathrm{Mg} / \mathrm{H}]_{\text {Sun }}=3.8 \times 10^{-5}$ (Sect. 4.2.1). The temperature is consistent with an upper limit derived from the $b$-parameter of the strongest $\mathrm{Mg}$ II line, $T \leq 13500 \mathrm{~K}$. We note that the narrow $\mathrm{Mg}_{\mathrm{I}}$ velocity component gives an upper temperature limit of $T \leq 6500 \mathrm{~K}$, or with a more conservative error interpretation, $T \leq 10500 \mathrm{~K}$ (Sect. 4.1.1).

Bergeron et al. (1994) presented a detailed two-phase photoionization model for the $z_{\mathrm{abs}}=0.79$ absorber toward PKS 2145+067. They used low resolution HST/FOS data covering several higher ionization lines of $\mathrm{Si}, \mathrm{C}$, and $\mathrm{O}$, and data of higher resolution for $\mathrm{Mg}$ II $\left(\cdot R \approx 10^{4}\right.$, three detected Mg II components: Petitjean \& Bergeron 1990). No measured $\mathrm{Mg}_{\mathrm{I}}$ and only an upper limit on Fe II were available. Bergeron et al. (1994) applied a power-law radiation field with $J_{v_{0}}^{z_{\text {abs }}=0.79}=1.3 \times 10^{-22} \mathrm{erg} \mathrm{s}^{-1} \mathrm{~cm}^{-2} \mathrm{~Hz}^{-1} \mathrm{sr}^{-1}$, a power-law in$\operatorname{dex} \beta=-0.5$, and a break at $54.4 \mathrm{eV}$ of a factor of 5 (Madau 1992). They also investigated a possible contribution by the absorbing galaxy to the UV flux, but with no significant change for the model results. Bergeron et al. (1994) find a surprisingly high $\mathrm{Mg}$ abundance $[\mathrm{Mg} / \mathrm{H}] \approx 1.2 \times 10^{-5}$, together with a temperature $T_{\mathrm{e}}=12450 \mathrm{~K}$ and a total hydrogen density $n_{\mathrm{H}}=5.7 \times 10^{-3} \mathrm{~cm}^{-3}$ in the low-ionization phase of $6.7 \mathrm{kpc}$ extent. The determined $\mathrm{Mg}^{+} / \mathrm{Mg}$ ratio is 165 . The calculated Fe II column density of $N=3.1 \times 10^{11} \mathrm{~cm}^{-2}$ is more than an order of magnitude lower than our measured value (cf. Table 3 ). The differences between our model and the model by Bergeron et al. (1994) have two main causes: radiation fields and atomic physics. Our radiation field is of roughly similar intensity and slope in the range 400-912 $\AA$, but steeper and about an order of magnitude more intense above $912 \AA$, and of much lower intensity below $400 \AA$ (cf. Table 4). Secondly, Bergeron et al. (1994) have used the rate coefficients adopted by Petitjean et al. (1992), who did not consider the $\mathrm{Mg}+\mathrm{H}^{+}$charge-transfer.

We conclude here that the contribution of the absorbing galaxy to the UV flux at the absorber and $\mathrm{Mg}+\mathrm{H}^{+}$ charge-transfer play significant roles in photoionization models of Mg II absorbers. The effects of significant host galaxy UV flux might partly explain inconsistencies in representations of $\mathrm{Mg}_{\text {II }}$ absorbers spread over redshifts $0.4 \lesssim z_{\mathrm{abs}} \lesssim$ 1.2 , which are based on photoionization models with a common radiation field for all absorbers: models seem not to reproduce the observed $\mathrm{Mg}$ I to $\mathrm{Mg}$ II column density ratios in many cases (Churchill et al. 2003). To resolve this issue, Ding et al. (2003) inferred the existence of a separate $\mathrm{Mg}_{\mathrm{I}}$ phase on the basis of a photoionization model for a strong Mg II absorber toward PG 1634+706. They propose that this phase gives rise to the bulk of the $\mathrm{Mg}$ I absorption in the form of very narrow components $\left(b<1 \mathrm{~km} \mathrm{~s}^{-1}\right)$. At the resolution of their Keck/HIRES spectra, $R \approx 45000$ or $6.6 \mathrm{~km} \mathrm{~s}^{-1}$, they could not obtain a unique Voigt profile fit to the weak, blended $\mathrm{Mg}$ I profile. In the case of PKS $2145+067$, there seems to be only a single, unblended $\mathrm{Mg}$ I component (cf. Fig. 2). At a resolution of $R \approx 100000$ or $3 \mathrm{~km} \mathrm{~s}^{-1}$, we measure a small Doppler parameter for $\mathrm{Mg}_{\mathrm{I}}$ of $b \approx 1 \mathrm{~km} \mathrm{~s}^{-1}$. However, the $\mathrm{Mg}_{\mathrm{I}}$ Doppler parameter is not significantly smaller than typical $b$ values of 3-4 $\mathrm{km} \mathrm{s}^{-1}$ seen for $\mathrm{Mg}$ II, especially considering the measurement uncertainty (cf. Table 3). Without a more detailed model, on the basis of the narrow observed line width alone, we cannot draw a reliable conclusion here.

For detailed photoionization models of both low- and highionization phases, HST/STIS observations of higher ionization species in combination with high-resolution data for $\mathrm{Mg}$ II, $\mathrm{Mg}_{\mathrm{I}}, \mathrm{Fe}$ II, and other ions are desirable (see e.g., Charlton et al. 2000; Ding et al. 2003). In the case of PKS 2145+067, HST/STIS observations of the quasar and the absorber host galaxy would be a valuable complement to our high-resolution data: measurement of the UV flux escaping from the host galaxy and analysis of the higher ionization species with higher resolution and better accuracy.

Acknowledgements. This research was supported by "Vetenskapsrådet" (VR), the Swedish Research Council. A.T. thanks VR for the financial support during the time 1999-2003 (Ph.D. grant 2002-685). We are grateful to ESO and the Paranal Observatory staff for efficiently conducting the reported VLT service-mode observations. 


\section{References}

Abazajian, K., Adelman-McCarthy, J. K., Agüeros, M. A., et al. 2003, AJ, 126, 2081

Allan, R. J., Clegg, R. E. S., Dickinson, A. S., \& Flower, D. R. 1988, MNRAS, 235, 1245

Bahcall, J. N., Bergeron, J., Boksenberg, A., et al. 1993, ApJS, 87, 1

Bahcall, J. N., Steinhardt, C. L., \& Schlegel, D. 2004, ApJ, 600, 520

Baldwin, J. A. 1977, ApJ, 214, 679

Barrow, J. D., Magueijo, J., \& Sandvik, H. B. 2002a, Phys. Rev. D, 66, 3515

Barrow, J. D., Sandvik, H. B., \& Magueijo, J. 2002b, Phys. Rev. D, 65,3504

Bechtold, J., Dobrzycki, A., Wilden, B., et al. 2002, ApJS, 140, 143

Bergeron, J., \& Boissé, P. 1991, A\&A, 243, 344

Bergeron, J., Petitjean, P., Sargent, W. L. W., et al. 1994, ApJ, 436, 33

Boivin, R. F., \& Srivastava, S. K. 1998, J. Phys. B, 31, 2381

Buat, V., Burgarella, D., Deharveng, J. M., \& Kunth, D. 2002, A\&A, 393, 33

Chand, H., Srianand, R., Petitjean, P., \& Aracil, B. 2004, A\&A, 417, 853

Charlton, J. C., Mellon, R. R., Rigby, J. R., \& Churchill, C. W. 2000, ApJ, 545, 635

Chen, H.-W., Lanzetta, K. M., Webb, J. K., \& Barcons, X. 2001, ApJ, 559,654

Churchill, C. W., \& Vogt, S. S. 2001, AJ, 122, 679

Churchill, C. W., Vogt, S. S., \& Charlton, J. C. 2003, AJ, 125, 98

Cooke, A. J. 1994, Ph.D. Thesis, University of Cambridge

Ding, J., Charlton, J. C., Bond, N. A., Zonak, S. G., \& Churchill, C. W. 2003, ApJ, 587, 551

Dzuba, V. A., Flambaum, V. V., \& Webb, J. K. 1999a, Phys. Rev. A, 59,230

Dzuba, V. A., Flambaum, V. V., \& Webb, J. K. 1999b, Phys. Rev. Lett., 82,888

Fan, X., Strauss, M. A., Schneider, D. P., et al. 2003, AJ, 125, 1649

Ferland, G. J. 2003, ARA\&A, 41, 517

Haardt, F., \& Madau, P. 1996, ApJ, 461, 20

Kayser, R., Helbig, P., \& Schramm, T. 1997, A\&A, 318, 680

Lotz, W. 1967, ApJS, 14, 207

Loveday, J., Peterson, B. A., Efstathiou, G., \& Maddox, S. J. 1992, ApJ, 390, 338
Madau, P. 1992, ApJ, 389, L1

Mendoza, C., \& Zeippen, C. J. 1987, A\&A, 179, 346

Morton, D. C. 1991, ApJS, 77, 119

Morton, D. C. 2003, ApJS, 149, 205

Murphy, M. T., Webb, J. K., Flambaum, V. V., Churchill, C. W., \& Prochaska, J. X. 2001a, MNRAS, 327, 1223

Murphy, M. T., Webb, J. K., Flambaum, V. V., et al. 2001b, MNRAS, 327, 1208

Murphy, M. T., Webb, J. K., \& Flambaum, V. V. 2003a, MNRAS, 345, 609

Murphy, M. T., Webb, J. K., Flambaum, V. V., \& Curran, S. J. 2003b, Ap\&SS, 283, 577

Nussbaumer, H., \& Storey, P. J. 1986, A\&AS, 64, 545

Osmer, P. S., \& Shields, J. C. 1999, in Quasars and Cosmology, ed. G. Ferland, \& J. Baldwin (San Francisco: ASP), ASP Conf. Ser., 162,235

Osterbrock, D. E., \& Mathews, W. G. 1986, ARA\&A, 24, 171

Petitjean, P., \& Bergeron, J. 1990, A\&A, 231, 309

Petitjean, P., Bergeron, J., \& Puget, J. L. 1992, A\&A, 265, 375

Pogge, R. W., \& Peterson, B. M. 1992, AJ, 103, 1084

Quast, R., Reimers, D., \& Levshakov, S. A. 2004, A\&A, 415, L7

Sandvik, H. B., Barrow, J. D., \& Magueijo, J. 2002, Phys. Rev. Lett., 88,1302

Schneider, D. P., Fan, X., Hall, P. B., et al. 2003, AJ, 126, 2579

Scott, J., Bechtold, J., Morita, M., Dobrzycki, A., \& Kulkarni, V. P. 2002, ApJ, 571, 665

Steidel, C. C., \& Sargent, W. L. W. 1991, ApJ, 382, 433

Steidel, C. C., \& Sargent, W. L. W. 1992, ApJS, 80, 1

Steidel, C. C., Kollmeier, J. A., Shapley, A. E., et al. 2002, ApJ, 570, 526

Sulentic, J. W., Marziani, P., \& Dultzin-Hacyan, D. 2000, ARA\&A, 38,521

Tappe, A., \& Black, J. H. 2004, in preparation

Véron-Cetty, M. P., \& Véron, P. 2000, A\&AR, 10, 81

Webb, J. K. 1987, Ph.D. Thesis, University of Cambridge

Webb, J. K., Murphy, M. T., Flambaum, V. V., et al. 2001, Phys. Rev. Lett., 87, 1301

Webb, J. K., Murphy, M. T., Flambaum, V. V., \& Curran, S. J. 2003, Ap\&SS, 283, 565

Weisheit, J. C. 1978, ApJ, 219, 829 